\title{
A autocompaixão em mulheres e relações com autoestima, autoeficácia e aspectos sociodemográficos*
}

\author{
Luciana Karine de Souza \\ Universidade Federal do Rio Grande do Sul, RS, Brasil, \\ Universidade Federal de Minas Gerais, MG, Brasil \\ Claudio Simon Hutz \\ Universidade Federal do Rio Grande do Sul, RS, Brasil
}

\begin{abstract}
Resumo
Estudos mostram que mulheres são mais acometidas por sintomas de depressão e ansiedade, na comparação com homens. Uma das maneiras de se contrabalançar tais sintomas é através da autocompaixão. Em estudo brasileiro prévio, mulheres apresentaram menor autocompaixão do que homens. O presente estudo investigou diferenças na autocompaixão em 327 mulheres com respeito à autoestima, autoeficácia e aspectos sociodemográficos. As participantes responderam um questionário sociodemográfico e escalas de autocompaixão, autoestima e autoeficácia. Comparações etárias e com variáveis sociodemográficas foram realizadas mediante testes t. Os resultados significativos encontrados para autocompaixão foram ter mais de 30 anos, praticar uma religião, não usar medicamento psiquiátrico e ter filhos. O trabalho permitiu a identificação de variáveis que sugerem direções para futuras pesquisas sobre autocompaixão em mulheres. Um exemplo é a questão da relação entre ter filhos e maior autocompaixão, o que pode sugerir que habilidades maternas e estilos parentais possam desempenhar papel importante nessa questão.
\end{abstract}

Palavras-chave: Compaixão; Autoavaliação; Autoestima; Autoeficácia; Mulheres.

\section{Self-compassion in brazilian women and relations with self-esteem, self-efficacy and demographic aspects}

\begin{abstract}
Studies show that women, compared to men, are more affected by symptoms of depression and anxiety. One way to counteract these symptoms is by self-compassion. In a previous Brazilian study, women showed lower self-compassion than men. The present study investigated differences in self-compassion in 327 women with regard to self-esteem, self-efficacy and sociodemographic aspects. The participants answered a sociodemographic questionnaire and self-compassion, self-esteem and self-efficacy scales. $\mathrm{T}$ tests examined age and sociodemographic differences. The significant differences found for self-compassion were: be over 30 years of age, to practice a religion, not use psychiatric medication and have children. This study allowed for the identification of variables that suggest directions for further research about self-compassion in women. One example is the question of the relationship between having children and greater self-compassion, which may suggest that maternal skills and parenting styles can play an important role in this matter.
\end{abstract}

Keywords: Compassion; Self-evaluation; Self-esteem; Self-efficacy; Women.

\section{La autocompasión entre las mujeres brasileñas y las relaciones con la autoestima, la autoeficacia y aspectos demográficos}

\section{Resumen}

Los estudios demuestran que las mujeres se ven más afectadas por los síntomas de depresión y ansiedad, en comparación con los hombres. Una forma de contrarrestar estos síntomas es por la autocompasión. En estudio brasileño anterior, las mujeres tenían menor autocompasión que los hombres. Este estudio investigó las diferencias en la autocompasión en 327 mujeres con respecto a la autoestima, la autoeficacia y aspectos demográficos. Los participantes respondieron un cuestionario escalas y la autocompasión sociodemográficas, la autoestima y la autoeficacia. Comparaciones edad y las variables sociodemográficas se realizó mediante pruebas t. Se encontró que los resultados significativos de la autocompasión tener más de 30 años, la práctica de una religión, no use la medicación psiquiátrica y tener hijos. El trabajo permitió la identificación de las variables que sugieren direcciones para la investigación futura en la autocompasión en las mujeres. Un ejemplo es la cuestión de la relación entre tener hijos y una mayor autocompasión, lo que puede sugerir que las habilidades maternas y los estilos de crianza pueden jugar un papel importante en este tema.

Palabras-clave: Compasión; Autoevaluación; Autoestima; Autoeficacia; Mujeres.

\footnotetext{
*Este estudo foi realizado com amostra advinda da coleta de dados da pesquisa “Adaptação e Validação da Escala de Autocompaixão", desenvolvida nos estudos de pós-doutorado da primeira autora, sob supervisão do segundo autor, com apoio financeiro do CNPq (projeto 159687/2011-0). Apoio institucional Departamento de Psicologia da Universidade Federal de Minas Gerais e Programa de Pós-Graduação em Psicologia da Universidade Federal do Rio Grande do Sul (UFRGS). Agradecimentos: K. Neff, L. Taborda, J. Natividade, H. Fernandes, J. Rique, A. Antoniazzi, J. Sarriera, e a equipe de 2012 do Laboratório de Mensuração da UFRGS.
} 
Estudos têm mostrado que mulheres são mais acometidas por sintomas de depressão e ansiedade do que homens (Brandtner \& Bardagi, 2009; Correia \& Borloti, 2011; Justo \& Calil, 2006; Rombaldi, Silva, Gazalle, Azevedo \& Hallal, 2010). No Brasil, a última Pesquisa Nacional de Saúde (Instituto Brasileiro de Geografia e Estatística, 2014) aponta maior prevalência de depressão em mulheres $(10,9 \%)$ na comparação com homens $(3,9 \%)$ (p.50).

Uma das maneiras de se contrabalançar sintomas depressivos e de ansiedade é através da autocompaixão. A autocompaixão envolve seis atitudes interrelacionadas diante de experiências de sofrimento, erros e fracassos: ser bondoso consigo, evitar autocrítica severa, ser mindful (consciência equilibrada dos próprios pensamentos e sentimentos), não se identificar com nem negar o sofrimento, não se isolar das outras pessoas, e compreender que o sofrimento é parte das experiências de qualquer ser humano (Neff, 2003a, 2003b).

Com a autocompaixão, há o entendimento de que o sofrimento (erro, fracasso, dor, inadequação) é parte da experiência humana, compartilhada, e que, portanto, não cabe absorver o eu como se fosse o único a passar por experiências negativas. Age-se com bondade e compreensão consigo, de forma serena e atenta aos pensamentos e sentimentos negativos oriundos da experiência de sofrimento, com o automonitoramento de não se deixar absorver por ela, nem a negar, e nem se avaliar negativamente com severidade. Trata-se de alcançar um equilíbrio mental (conjuntamente cognitivo e afetivo) que não permite que o eu se deixe reduzir a ponto de ser tomado por todo o sofrimento, nem de sobrecarrega-lo com preocupações e ruminações por angústia que precede, ou sucede, eventos negativos.

A autocompaixão é um construto da saúde mental. Estudos mostram que elevada autocompaixão está associada com menor depressão, ansiedade, raiva, sentimentos negativos, autocrítica, fracasso acadêmico e estresse (Neely, Schallert, Mohammed, Roberts \& Chen, 2009; Neff, Hsieh \& Dejitterat, 2005; Raes, 2011; Shapira \& Mongrain, 2010), e com maior tomada de perspectiva, otimismo, autoestima, perdão, empatia e qualidade de vida (Neff \& Pommier, 2013; Neff \& Vonk, 2009; Van Dam, Sheppard, Forsyth \& Earleywine, 2011; Wei, Liao, Ku \& Shaffer, 2011). De fato, intervenções que promoveram atitudes autocompassivas diminuíram índices de depressão e ansiedade (Raes, 2011; Shapira \& Mongrain, 2010). A autocompaixão está presente no universo de construtos positivos estudados pelo movimento da psicologia positiva, como mostram Neff e Lamb (2009).
Já o primeiro estudo em autocompaixão indicou menores índices em mulheres (Neff, 2003a). Recentemente, Yarnell, Stafford, Neff, Reilly, Knox e Mullarkey (2015) conduziram uma metanálise com 71 pesquisas sobre autocompaixão e sexo. O resultado confirmou a menor autocompaixão em mulheres, ainda que a diferença encontrada tenha sido de pequeno efeito $(\mathrm{d}=0,18)$. No Brasil, o único estudo sobre autocompaixão conduzido até então também encontrou maior autocompaixão em homens, com efeito de 0,25 (Souza \& Hutz, 2016a).

Os dados mencionados sugerem maior atenção à autocompaixão em mulheres e sua relação com outras variáveis. Neste caminho, poder-se-ia detectar diferenças entre mulheres na busca por maior especificação da menor autocompaixão em amostras femininas.

Contudo, a bibliografia disponível especificamente dedicada à autocompaixão em mulheres é pequena. Uma busca por "self-compassion AND women" e por "self-compassion AND woman" no site Periódicos CAPES em abril de 2015 localizou apenas nove artigos empíricos. Um panorama conciso sobre esta literatura é oferecido a seguir.

Arch, Brown, Dean, Landy, Brown e Laudenslager (2014) avaliaram respostas biopsicológicas (saliva, batimentos cardíacos e comportamentos indicadores de ansiedade) para uma tarefa que provocava estresse social. Foi oferecido um curto treinamento em autocompaixão para um grupo de mulheres, nenhum treinamento para um segundo grupo, e uma "ação placebo" para um terceiro. O treinamento em autocompaixão diminuiu os índices avaliados, com exceção do cortisol, e as participantes com maior autocompaixão apresentaram menor sensação de ameaça social. Dessa forma, a autocompaixão se mostra relevante diante de sintomas de ansiedade social ou circunstâncias ameaçadoras ao eu em contextos de relações interpessoais que posicionem o sujeito em alguma situação de comparação social desvantajosa.

Focalizando a autoimagem, Wasylkiw, MacKinnon e MacLellan (2012) a estudaram na relação com a autocompaixão em 187 mulheres. Índices elevados de autocompaixão foram capazes de predizer menor preocupação com o corpo e culpa ao comer, a despeito da autoestima. Na inclusão conjunta da autocompaixão e autoestima como preditoras no modelo, foi a autocompaixão que respaldou melhor na porcentagem da variância na preocupação corporal e com o peso. Finalmente, a autocompaixão foi responsável por mediar parcialmente a relação entre preocupação com o corpo e sintomas depressivos. Liss e Erchull (2015) observaram, em 306 mulheres, que a autocompaixão é maior quando menores é a vigilância corporal, 
vergonha corporal, depressão e atitudes negativas com relação ao comer. $\mathrm{O}$ estudo sugere que mulheres em risco para preocupações com o peso, o corpo e o comer com culpa podem se beneficiar da autocompaixão preventivamente.

Voltada para o conceito de alimentação intuitiva (rejeitar modas de dietas, comer quando sente fome, atentar para a saciedade e a satisfação, exercitar, respeitar o próprio corpo) a pesquisa de Schoenefeld e Webb (2013) buscou aproximações com a autocompaixão em 322 estudantes universitárias. As análises mostraram uma associação positiva e significativa entre o comer intuitivo e a autocompaixão. A aceitação da imagem corporal colaborou para explicar essa associação.

No quarto estudo localizado sobre autocompaixão em mulheres no contexto da alimentação, Daye, Webb e Jafari (2014) argumentam que há relatos de que cuidadores, na preocupação de garantir a alimentação de seus pacientes, exageram na pressão para que estes comam, gerando sentimentos negativos nestes últimos, alguns dos quais relacionados à autoimagem corporal. Maiores índices de autocompaixão se associaram com menor vergonha e vigilância do corpo. Além disso, a autocompaixão se mostrou capaz de atenuar a relação entre mensagens críticas de cuidadores sobre comer, e a vergonha e vigilância corporais.

Quatro pesquisas sobre autocompaixão em mulheres se dedicaram a atletas. Mosewich, Kowalski, Sabiston, Sedgwick e Tracy (2011) encontraram, em 151 mulheres atletas, que a autocompaixão se correlacionou negativamente com tendência a sentir vergonha, ansiedade quanto ao físico, medo do fracasso, medo de avaliações negativas e consciência do corpo como um objeto. Os autores entendem que a promoção da autocompaixão pode cultivar experiências esportivas positivas em mulheres atletas.

Mosewich, Crocker, Kowalski e Delongis (2013) observaram, em 29 atletas femininas, que aquelas com menor autocompaixão se beneficiaram de um programa para promover autocompaixão, pois obtiveram menores escores em autocrítica, ruminação e preocupação com erros. Nesta mesma linha experimental, Reis, Kowalski, Ferguson, Sabiston, Sedgwick e Crocker (2015) verificaram que uma intervenção breve para promover autocompaixão obteve resultados limitados em 21 atletas. Ainda assim, as avaliações de autocompaixão, autoestima, narcisismo e reações a cenários hipotéticos de derrota e fracasso, prévias e posteriores à intervenção, mostraram que o aumento da autocompaixão elevava as respostas saudáveis aos cenários desafiadores. Acima de tudo, os benefícios da autocompaixão foram maiores do que os da autoestima e do narcisismo.
Ferguson, Kowalski, Mack e Sabiston (2014) identificaram uma correlação positiva e significativa entre autocompaixão e bem-estar em 83 mulheres. Testes mostraram que autocompaixão, resistência passiva, responsabilidade, iniciativa e autodeterminação responderam por $83 \%$ da variância no bem-estar. Em seguida, um estudo qualitativo com 11 atletas revelou que a autocompaixão é vantajosa em situações esportivas difíceis ao aumentar a positividade, a perseverança e a responsabilidade e reduzir a ruminação.

Como visto, a literatura publicada disponível sobre autocompaixão em amostras femininas é ainda escassa, mas indicando implicações da autocompaixão para comportamentos saudáveis na alimentação e nos esportes. Os estudos mostraram associação entre menor autocompaixão e maior preocupação e, portanto, ansiedade, bem como depressão, com relação ao comer e à imagem corporal. Da mesma forma, foram positivas as associações entre maior autocompaixão e melhor desempenho em mulheres atletas.

$\mathrm{Se}$ as mulheres são propensas a menor autocompaixão, intervir para promovê-la tem seu valor não somente para ajudar a superarem desafios e dificuldades, mas também para incentivá-las a perseguir suas vocações no esporte e no atletismo. Da mesma forma, estudos mostram que a preocupação com a forma física, a magreza e o peso têm levado mulheres a desenvolverem hábitos alimentares não-saudáveis. A promoção da autocompaixão pode servir como um contrabalanço em relação à tendência à desvalorização da própria imagem. Em comum, os nove estudos revisados demonstram a forte relação entre autocompaixão e sintomas depressivos e ansiosos em mulheres, o que fortalece o entendimento da autocompaixão como um construto de saúde psicológica.

Medidas de autoestima e de autoeficácia são tradicionalmente mais utilizadas para a avaliação global de capacidades do eu para lidar consigo e com experiências da vida. Na medida de autoestima de Rosenberg, bastante empregada em pesquisa, é considerada a atitude com relação à imagem corporal (Hutz \& Zanon, 2011). Na avaliação da autoeficácia, isto é, a crença estável na capacidade de se alcançar os objetivos, estão incluídos itens que lidam com desafios e dificuldades - experiências alvo da atitude autocompassiva. Portanto, estudos que contemplem a autocompaixão podem se beneficiar da inclusão de medidas mais comuns direcionadas ao eu, como é o caso da autoestima e da autoeficácia. E possivelmente há outros aspectos que podem ser investigados com respeito à autocompaixão em mulheres.

O presente estudo buscou investigar diferenças na autocompaixão em mulheres com respeito à autoestima, 
autoeficácia e aspectos sociodemográficos, dentre os quais a faixa etária, estado civil, prática religiosa e uso de terapia. Trata-se de um estudo exploratório que busca contribuir com o conhecimento acerca da autocompaixão em mulheres.

\section{Método}

\section{Participantes}

Participaram 327 mulheres que não foram sorteadas para compor a amostra do estudo de adaptação e validação da Escala de Autocompaixão (Souza \& Hutz, 2016a). A faixa etária variou de 18 a 71 anos, com média de 32,7 $(\mathrm{DP}=10,9)$. Aproximadamente metade das participantes estavam na faixa etária de 18 a 30 anos $(52,5 \%)$. As participantes eram residentes de 18 diferentes estados brasileiros e Distrito Federal, com $29 \%$ de participação oriunda do Rio Grande do Sul, $26 \%$ de Minas Gerais e 23\% de São Paulo.

\section{Instrumentos}

Os instrumentos utilizados na coleta de dados, todos de autorrelato, foram: um questionário sociodemográfico de uma página (sexo, idade, cidade de residência, estado civil, filhos, atividade remunerada, nível educacional, religião e prática, uso de medicação psiquiátrica e experiência com psicoterapia). Em seguida, as participantes preencheram a Escala de Autocompaixão, a Escala de Autoeficácia e a Escala de Autoestima de Rosenberg. Para nenhum instrumento foi solicitado o nome do participante. O tempo total de preenchimento varia entre 10 e 20 minutos.

A Escala de Autocompaixão foi desenvolvida por Neff (2003b) e é composta por 26 itens (cada qual avaliado por uma escala Likert de cinco pontos) que agregam as facetas de isolamento, senso de humanidade, mindfulness, sobreidentificação, bondade consigo e autocrítica severa. A escala original apresentou um coeficiente alpha de 0,92 . A adaptação e validação de construto da escala para uso no Brasil foi realizada por Souza e Hutz (no prelo, a) e apresentou o mesmo índice de consistência interna. A Escala de Autoeficácia foi criada para uso com brasileiros por Pacico, Ferraz e Hutz (2014) a partir de escalas disponíveis e do estudo das dimensões envolvidas na teoria que fundamenta o entendimento do conceito. Possui 20 itens (escala Likert de cinco pontos) e a consistência interna é de 0,89. São exemplos de itens "Eu costumo persistir em meus planos" e "Sou uma pessoa autoconfiante". A Escala de Autoestima de Rosenberg foi adaptada e validada por Hutz e Zanon (2011) para uso no Brasil, possui 10 itens (escala Likert de quatro pontos), e a consistência interna foi de 0,90 .

\section{Procedimentos de coleta de dados}

Após aprovação da pesquisa principal no comitê de ética da universidade (CAAE 04345112.5.0000.5334), o Termo de Consentimento Livre e Esclarecido (TCLE) e os instrumentos (na ordem supracitada) foram inseridos na plataforma surveymonkey. Criouse um link para a pesquisa, com um convite no corpo de uma mensagem eletrônica, amplamente divulgada pela Internet (via mensagens de e-mail). Clicando no link, o participante era levado para a página do TCLE ao final do qual se pedia para clicar em "concordo" ou "não concordo" em participar da pesquisa: clicando no primeiro, o respondente era levado para a primeira página de instrumentos; clicando no segundo, aparecia uma mensagem de agradecimento e despedida.

No fechamento da coleta de dados, após 30 dias, aproximadamente $70 \%$ dos respondentes da pesquisa eram mulheres e, após o sorteio de uma mesma quantidade de participantes homens e mulheres para o estudo de validação da Escala de Autocompaixão, restaram, ainda, 327 casos válidos de respondentes do sexo feminino. Este banco restante exclusivamente feminino motivou o presente trabalho.

\section{Análise dos dados}

Em primeiro lugar, foram descartados os casos sem identificação de sexo, idade ou outra variável de interesse do estudo. Dessa forma, todas as 327 participantes responderam a todos os campos dos instrumentos da pesquisa. Apenas os autores tiveram acesso ao banco de dados para análise. A análise dos dados foi realizada com auxílio do software SPSS versão 21. Primeiro, foram geradas estatísticas descritivas simples. Em seguida, correlações de Pearson foram obtidas entre as medidas de autocompaixão, autoestima e autoeficácia. O terceiro passo da análise envolveu cálculos de testes $\underline{\mathrm{t}}$ para comparações de grupos conforme sexo, faixa etária (grupos 18-30 anos e 31-71 anos), religião e sua prática, experiência com psicoterapia, uso de medicamento psiquiátrico, ter atividade remunerada e filhos. O tamanho do efeito via $\underline{\mathrm{d}}$ de Cohen foi calculado para as comparações de grupos. A significância mínima requerida para a obtenção de resultados estatisticamente válidos foi de 0,05 .

\section{Resultados}

São apresentados primeiramente as frequências e porcentagens relativas aos dados sociodemográficos e variáveis coletadas no questionário que antecedeu as escalas. Em seguida, são exibidas as correlações entre as escalas e as comparações de grupos. 
Nos dados sociodemográficos, as frequências e porcentagens encontradas foram as seguintes: para faixa etária, foram 172 pessoas $(52,5 \%)$ de 18 a 30 anos de idade, e 155 (47,5\%) entre 31 e 71; no estado civil, 138 (42\%) de casados, 102 (31\%) de solteiros, 68 (21\%) participantes declararam estar namorando, e 12 (4\%) separados/divorciados; para religião, foram 169 (52\%) de católicos, 57 (17\%) de espíritas, 29 (9\%) de protestantes e $2(1 \%)$ de budistas; 183 praticam sua religião (56\%), 231 (71\%) têm experiência prévia com psicoterapia; 79 (24\%) fazem psicoterapia atualmente; 37 (11\%) usam medicamento psiquiátrico; 256 (78\%) exercem atividade remunerada; e 99 (30\%) possuem filhos.

Para o estado civil, foi necessário construir a categoria "outras respostas" $(\mathrm{n}=7)$, assim como outras respostas sobre religião $(n=3)$. As religiões protestantes foram agrupadas; separadas, foram citadas como: evangélica (maior frequência, com $n$ =12), luterana, protestante, batista e adventista do sétimo dia. Já as demais "outras religiões" citadas foram: cristã $(n=8)$, judaica, candomblé, umbandista, mórmon, neopaganismo e católica/espírita. Optou-se por destacar a religião budista pela sua proximidade com o conceito de autocompaixão conforme delineado por Neff (2003a). Foram 51 (16\%) as participantes que informaram não possuir religião ou serem ateias.

Aquestão "Possui ensino superior completo?" gerou uma série de resultados incompatíveis com o esperado. Muitas participantes de 18 e 19 anos responderam afirmativamente, o que não é congruente com tempo necessário para a realização e conclusão de um curso superior como Direito, por exemplo. Por esta razão, os dados sobre a escolaridade não foram aproveitados. De posse de todos os dados sociodemográficos brutos, uma representante da amostra coletada seria casada, praticante do catolicismo, sem filhos, exercendo atividade remunerada, com experiência anterior em psicoterapia, sem consultar atualmente e sem usar medicamento psiquiátrico.
As correlações encontradas entre as escalas aplicadas foram: autocompaixão com autoestima a $\mathrm{r}=0,69$ e com autoeficácia a $\mathrm{r}=0,53$, e autoestima com autoeficácia a $r=0,68$. São correlações significativas $(\mathrm{p}<0,001)$ consideradas altas e que repetem o padrão encontrado em outros estudos (Neff \& Vonk, 2009; Souza \& Hutz, 2016a).

Foram conduzidas comparações de grupos com relação à autocompaixão. As diferenças significativas se localizaram na faixa etária maior, em não usar medicamento psiquiátrico, em praticar a religião e em ter filhos. A Tabela 1 apresenta estes resultados.

As comparações seguintes não mostraram diferenças estatisticamente significativas na amostra feminina estudada: experiência anterior com psicoterapia, experiência corrente com psicoterapia e exercer atividade remunerada. Não foram realizadas comparações com relação à religião porque a categoria "budista" foi marcada apenas por duas pessoas na amostra.

Interessantemente, na atividade remunerada foram significativas as comparações para autoestima e para autoeficácia. As participantes com maior autoestima e com maior senso de autoeficácia exerciam atividade remunerada. Em virtude disso, optou-se por relatar, de modo complementar aos propósitos deste trabalho, as médias das variáveis investigadas com relação à autoestima e à autoeficácia.

Nas comparações de grupos realizadas, as diferenças significativas para autoestima foram na faixa etária maior, em não usar medicamento psiquiátrico, em praticar a religião, em possuir atividade remunerada e em ter filhos. A Tabela 2 exibe os achados estatísticos.

Nas comparações quanto à autoeficácia, as diferenças significativas foram para maior faixa etária, praticar a religião, usar de medicamento psiquiátrico e ter atividade remunerada. Os resultados constam na Tabela 3.

TABELA 1

Comparações de médias para autocompaixão na faixa etária, medicação, religião e filhos

\begin{tabular}{|c|c|c|c|c|c|}
\hline & & $M(D P)$ & $t$ & $p$ & $d$ \\
\hline Faixa etária & $\begin{array}{l}18-30(\mathrm{n}=172) \\
31-71(\mathrm{n}=155)\end{array}$ & $\begin{array}{l}3,04(0,68) \\
3,35(0,68)\end{array}$ & $-4,08$ & $<0,001$ & 0,45 \\
\hline Usar medicamento psiquiátrico & $\begin{array}{l}\operatorname{sim}(n=37) \\
\text { não }(n=290)\end{array}$ & $\begin{array}{l}2,98(0,74) \\
3,21(0,68)\end{array}$ & 1,95 & 0,052 & 0,32 \\
\hline Praticar a religião & $\begin{array}{l}\operatorname{sim}(n=183) \\
\text { não }(n=144)\end{array}$ & $\begin{array}{l}3,29(0,68) \\
3,05(0,69)\end{array}$ & $-3,24$ & 0,001 & 0,35 \\
\hline Ter filho(s) & $\begin{array}{l}\operatorname{sim}(n=99) \\
\text { não }(n=228)\end{array}$ & $\begin{array}{l}3,37(0,71) \\
3,11(0,67)\end{array}$ & $-3,15$ & 0,002 & 0,37 \\
\hline
\end{tabular}

$\mathrm{M}=$ média; $\mathrm{DP}=$ desvio-padrão; $\mathrm{t}=$ teste $\mathrm{t} ; \mathrm{d}=\mathrm{d}$ de Cohen; $\mathrm{p}<0,005$. 
TABELA 2

Comparações de médias para autoestima na faixa etária, medicação, religião e filhos

\begin{tabular}{|c|c|c|c|c|c|}
\hline & & $M(D P)$ & $t$ & $p$ & $d$ \\
\hline Faixa etária & $\begin{array}{l}18-30(\mathrm{n}=172) \\
31-71(\mathrm{n}=155)\end{array}$ & $\begin{array}{l}3,14(0,59) \\
3,39(0,55)\end{array}$ & $-4,06$ & $<0,001$ & 0,43 \\
\hline Usar medicamento psiquiátrico & $\begin{array}{l}\operatorname{sim}(n=37) \\
\text { não }(n=290)\end{array}$ & $\begin{array}{l}3,07(0,61) \\
3,29(0,58)\end{array}$ & 2,09 & 0,037 & 0,36 \\
\hline Praticar a religião & $\begin{array}{l}\operatorname{sim}(n=183) \\
\text { não }(n=144)\end{array}$ & $\begin{array}{l}3,32(0,55) \\
3,19(0,62)\end{array}$ & $-2,02$ & 0,045 & 0,22 \\
\hline Atividade remunerada & $\begin{array}{l}\operatorname{sim}(n=256) \\
\text { não }(n=71)\end{array}$ & $\begin{array}{l}3,31(0,56) \\
3,10(0,64)\end{array}$ & $-2,67$ & 0,008 & 0,34 \\
\hline Ter filho(s) & $\begin{array}{l}\operatorname{sim}(\mathrm{n}=99) \\
\text { não }(\mathrm{n}=228)\end{array}$ & $\begin{array}{l}3,40(0,55) \\
3,20(0,59)\end{array}$ & $-2,81$ & 0,005 & 0,35 \\
\hline
\end{tabular}

$\mathrm{M}=$ média; $\mathrm{DP}=$ desvio-padrão; $\mathrm{t}=$ teste $\mathrm{t} ; \mathrm{d}=\mathrm{d}$ de Cohen; $\mathrm{p}<0,005$.

TABELA 3

Comparações de médias para autoeficácia na faixa etária, medicação, religião e trabalho

\begin{tabular}{|c|c|c|c|c|c|}
\hline & & $M(D P)$ & $t$ & $p$ & $d$ \\
\hline \multirow[t]{2}{*}{ Faixa etária } & $18-30(\mathrm{n}=172)$ & $3,37(0,58)$ & \multirow{2}{*}{$-3,07$} & \multirow{2}{*}{0,002} & \multirow{2}{*}{0,33} \\
\hline & $31-71(\mathrm{n}=155)$ & $3,56(0,55)$ & & & \\
\hline \multirow[t]{2}{*}{ Usar medicamento psiquiátrico } & $\operatorname{sim}(n=37)$ & $3,28(0,59)$ & \multirow{2}{*}{2,01} & \multirow{2}{*}{0,046} & \multirow{2}{*}{0,34} \\
\hline & não $(\mathrm{n}=290)$ & $3,48(0,56)$ & & & \\
\hline \multirow[t]{2}{*}{ Praticar a religião } & $\operatorname{sim}(n=183)$ & $3,52(0,53)$ & \multirow{2}{*}{$-2,27$} & \multirow{2}{*}{0,024} & \multirow{2}{*}{0,24} \\
\hline & não (n = 144) & $3,38(0,61)$ & & & \\
\hline \multirow[t]{2}{*}{ Atividade remunerada } & $\operatorname{sim}(n=256)$ & $3,51(0,53)$ & \multirow{2}{*}{$-3,19$} & \multirow{2}{*}{0,002} & \multirow{2}{*}{0,40} \\
\hline & não $(\mathrm{n}=71)$ & $3,27(0,66)$ & & & \\
\hline
\end{tabular}

$\mathrm{M}=$ média; $\mathrm{DP}=$ desvio-padrão; $\mathrm{t}=$ teste $\mathrm{t} ; \mathrm{d}=\mathrm{d}$ de Cohen; $\mathrm{p}<0,005$.

Os resultados significativos resultantes das comparações de médias permitem identificar as variáveis que se diferenciaram quanto à autocompaixão. Nas mulheres participantes, a autocompaixão é maior em mulheres com idade superior a 30 anos, que não usam medicação psiquiátrica, praticam sua religião e têm filhos. Em complemento, mostraram dados não antecipados sobre a autoestima e a autoeficácia em mulheres adultas após os 30 anos de idade, que praticam sua religião, não usam medicamento psiquiátrico, exercem atividade remunerada e têm filhos. Estas mulheres possuem maior índice de autoestima e de autoeficácia geral (esta com exceção de ter filhos).

Dessa forma, as análises respondem à questão norteadora delineada no presente estudo exploratório: quais aspectos sociodemográficos se destacam na relação com a autocompaixão em uma amostra feminina? A seguir, é apresentada a discussão dos achados com respaldo de literatura pertinente, limitações do estudo e indicações para futuras pesquisas.

\section{Discussão}

Os resultados significativos encontrados para a autocompaixão em mulheres foram ter mais de 30 anos, praticar uma religião, não usar medicamento psiquiátrico e ter filhos. Foi respondida, portanto, a pergunta de pesquisa sobre diferenças na autocompaixão em mulheres. Além disso, autocompaixão, autoestima e autoeficácia geral se mostraram correlacionadas de modo positivo e estatisticamente significativo, refletindo achados prévios da literatura (Neff \& Vonk, 2009; Souza \& Hutz, 2016a). Assim, crer nas próprias capacidades para lidar com problemas, desafios e erros, ser autocompassivo diante de sofrimentos e fracassos e valorizar a si são aspectos autoperceptivos saudáveis e complementares.

Encontrou-se maior autocompaixão em maior faixa etária. Porém, as diferenças etárias não têm encontrado respaldo firme nas pesquisas da autocompaixão. $\mathrm{O}$ estudo de Neff e Vonk (2009) encontrou correlação significativa entre autocompaixão e idade em uma 
amostra com faixa etária de 18 a 83 anos $(\mathrm{r}=0,24$; $\mathrm{p}<0,001$ ), embora o trabalho de Phillips e Ferguson (2013) não tenha detectado correlação significativa $(\mathrm{p}=0,08)$. Já Neff e McGehee (2010) não encontraram diferenças significativas entre adolescentes e adultos-jovens, e nem Neff e Pommier (2013) entre estudantes universitários e adultos. Contudo, pesquisa recente com brasileiros encontrou diferença significativa $(\mathrm{p}=0,001)$, com o grupo etário mais velho (31-66 anos) com maior autocompaixão (3,33, $\mathrm{DP}=0,72)$ que o outro grupo $(18-30$ anos $)(3,05$, $\mathrm{DP}=0,66)$ (Souza \& Hutz, 2016b). Os dados do presente estudo repetem estas diferenças e parecem contribuir para apontar para diferenças etárias que merecem investigações mais detalhadas com respeito à autocompaixão. Em uma amostra somente de mulheres, as diferenças etárias seguem significativas na autocompaixão, refletindo o resultado da única pesquisa brasileira prévia.

Com relação à autoestima, as mulheres mais velhas a apresentaram em maior índice. Twenge e Campbell (2001) conduziram uma metanálise com estudos que utilizaram a Escala de Autoestima de Rosenberg, a mesma usada neste estudo, e encontraram diferenças etárias, com um crescendo da autoestima com o avanço etário. Já para a autoeficácia geral, não foram localizados estudos que especificamente apontassem para esta relação positiva entre idade e crença generalizada de autoeficácia. Contudo, as correlações encontradas com a autoestima e a autocompaixão sugerem que estudos com a autoeficácia geral mostrassem seu aumento com a idade, o que de fato se observou no presente estudo.

A diferença significativa encontrada na autocompaixão com respeito à prática religiosa, sendo aquela maior em quem pratica sua religião, não surpreende. De fato, estudos têm indicado que praticar a religião contribui substancialmente para o bem-estar psicológico (Joshi, Kumari \& Jain, 2008). Um exemplo é o de Maltby, Lewin e Day (1999), que identificaram que a prática específica de rezar foi o fator da religiosidade que mais contribuiu para o bem-estar psicológico. Bagley e Mallick (1997) compararam participação religiosa e autoestima em adolescentes de uma escola pública laica e de uma escola católica, e encontraram que, a despeito do tipo de escola, o aumento da autoestima era acompanhado pelo aumento na participação religiosa $(\mathrm{p}<0,05)$. Com respeito à autoeficácia geral, Byrne (2012) identificou uma relação positiva e significativa entre este construto e a religiosidade intrínseca, alertando para que futuras pesquisas sobre o papel da religiosidade na saúde considerem-na separadamente quanto a suas dimensões extrínseca e intrínseca. A religiosidade intrínseca posiciona a religião como um fim em si mesma, ao passo que a extrínseca a utiliza como um meio para atingir fins almejados. Portanto, os resultados relatados no presente trabalho são congruentes com estudos anteriores que contemplaram prática religiosa e medidas de autoavaliação, como no caso da autoestima e da autoeficácia geral. Para a autocompaixão, o rationale delineado na presente pesquisa também permite concluir que o resultado encontrado é congruente com o construto autocompaixão.

Com relação ao uso de medicamento psiquiátrico, pensava-se em investigar sua relação com a autocompaixão em virtude de que a medicação auxilia na modificação do estado de humor do sujeito, o que deve influenciar a forma de pensar sobre si e, portanto, sobre suas vivências, inclusive as negativas. Nenhum estudo foi localizado sobre a relação entre autocompaixão e medicação psiquiátrica. No entanto, o trabalho de Bertoldi, Barros, Hallal e Lima (2004), com 3182 adultos residentes na cidade gaúcha de Pelotas, mostrou que o grupo de medicamentos voltados ao sistema nervoso central apresentou a quarta maior porcentagem de medicamentos usados (10\%). Não foram calculadas diferenças para mulheres e homens por grupo medicamentoso, mas nos resultados gerais foi apontado que as mulheres parecem estar mais sujeitas à medicalização posto que mostraram uso muito superior, e estatisticamente significativo, ao dos homens.

Não há estudos disponíveis sobre autocompaixão, autoestima ou autoeficácia geral em mulheres com e sem filhos. Todavia, a pesquisa de Jeffries e Konnert (2002) sugere melhores índices de bem-estar em mulheres sem filhos por opção. Estas apresentaram maior bem-estar psicológico e maior senso de autonomia do que as mulheres que não podem ter filhos. Por outro lado, há correlações significativas, relatadas na literatura, entre bem-estar psicológico e autocompaixão (Neff \& McGehee, 2010); da mesma forma, entre autocompaixão e autonomia (Neff, 2003b). Ainda assim, o trabalho dos autores focalizou mulheres na meia-idade, ao passo que no presente estudo metade da amostra possui de 31 a 71 anos. Portanto, apenas estudos mais específicos poderão sinalizar o que pode estar relacionado à elevada autocompaixão, autoestima e autoeficácia geral em mulheres com filhos, na comparação com aquelas sem filhos. Será interessante comparar mulheres sem filhos por opção com mulheres com filhos, de faixa etária semelhante, quanto à autocompaixão, autoestima e autoeficácia.

Outro aspecto que pode estar associado a ter filhos e maior autocompaixão diz respeito a habilidades maternas ou estilos parentais. Nesse contexto, a 
autocompaixão está circundada por desafios relacionados às dificuldades, erros, inadequações e fracassos percebidos com relação a criar e cuidar dos filhos. Ela vai envolver "evitar culpar a si quando as metas parentais não são alcançadas, o que pode permitir um reengajamento na busca dessas metas" (Duncan, Coatsworth \& Greenberg, 2009, p.260). Além disso, a autocompaixão também pode diminuir reduzir as ameaçadoras críticas que os pais temem receber dos outros sobre suas habilidades parentais ou sobre o comportamento da criança. Enfrentar tais desafios coloca o sujeito diante da necessidade de utilizar boas estratégias cognitivas, emocionais e comportamentais. Uma maior autocompaixão auxilia nestas estratégias mais adequadas. Pesquisadores nos temas da parentalidade podem coletar dados envolvendo a autocompaixão e buscar evidências com participantes brasileiros.

Sobre a relação entre autocompaixão e atividade remunerada, não foram encontradas diferenças significativas. No entanto, o estudo de Abaci e Arda (2013) sugere aproximações entre as variáveis. Estes autores investigaram a relação entre autocompaixão e satisfação com o emprego em trabalhadores (65\% homens), com média de idade de 33,6 anos, e localizaram uma correlação positiva significativa moderada, segundo eles $(r=0,44 ; p<0,01)$. Não foram encontradas diferenças de sexo. Já com respeito à autoestima e à autoeficácia geral, Judge e Bono (2001) averiguaram correlações entre autoestima, autoeficácia geral, lócus de controle e estabilidade emocional (via baixo neuroticismo) com satisfação com o emprego.
Os autores identificaram correlações positivas e significativas de satisfação com o emprego e autoestima $(r=0,26)$ e autoeficácia geral $(r=0,45)$. Concluíram que estes dois construtos são fundamentais para avaliar satisfação no trabalho. Portanto, ainda que no presente estudo não tenha sido investigada a satisfação no trabalho, há uma diferença significativa entre mulheres que possuem atividade remunerada e maior índice de autocompaixão, autoestima e autoeficácia geral. O que faltaria averiguar, nesta relação, é a questão da satisfação com essa atividade geradora de renda.

\section{Considerações finais}

Como visto, enquanto estudo exploratório o presente trabalho não possuía hipóteses definidas. Havia apenas um estudo brasileiro prévio com a Escala de Autocompaixão. Dadas as questões postuladas com relação à menor autocompaixão em mulheres, pensouse no presente estudo com o intuito de identificar variáveis relevantes para futuros trabalhos. Estes, por sua vez, podem investigar melhor as variáveis abordadas, especificando-as. De fato, o trabalho permitiu a identificação de variáveis que sugerem direções para futuras pesquisas com mulheres no Brasil. Um exemplo é a questão da relação entre ter filhos e maior autocompaixão. Seria algo relacionado às habilidades maternas para lidar com a criação dos filhos? Futuros trabalhos podem se dedicar mais a aspectos como este, enriquecendo a linha de pesquisa em autocompaixão no país.

\section{Referências}

Abaci, R. \& Arda, D. (2013). Relationship between self-compassion and job satisfaction in white-collar workers. Procedia - Social and Behavioral Sciences, 106, 2241-2247. http://dx.doi.org/10.1016/j.sbspro.2013.12.255

Arch, J., Brown, K., Dean, D., Landy, L., Brown, K., \& Laudenslager, M. (2014). Self-compassion training modulates alpha-amylase, heart rate variability, and subjective responses to social evaluative threat in women. Psychoneuroendocrinology, 42, 49-58. http://dx.doi.org/10.1016/j.psyneuen.2013.12.018

Bagley, C. \& Mallick, K. (1997). Self-esteem and religiosity: comparison of 13- to 15-year-old students in catholic and public junior high schools. Canadian Journal of Education, 22(1), 89-92. Recuperado de $<\mathrm{http}$ ://www.csse-scee.ca/ CJE/Articles/FullText/CJE22-1/CJE22-1-Bagley.pdf. http://dx.doi.org/10.2307/1585814>.

Bertoldi, A., Barros, A., Hallal, P., \& Lima, R. (2004). Utilização de medicamentos em adultos: prevalência e determinantes individuais. Revista de Saúde Pública, 38(2), 228-238. http://dx.doi.org/10.1590/S0034-89102004000200012

Brandtner, M. \& Bardagi, M. (2009). Sintomatologia de depressão e ansiedade em estudantes de uma universidade privada do Rio Grande do Sul. Gerais: Revista Interinstitucional de Psicologia, 2(2), 81-91. Recuperado de $<\mathrm{http}: / /$ pepsic.bvsalud.org/pdf/gerais/v2n2/v2n2a04.pdf>.

Byrne, P. (2012). I believe; I can. The relationship between religiosity, general self-efficacy and locus of control. Submitted in partial fulfilment of the requirements for the Higher Diploma in Psychology at Dublin Business School of Arts, Dublin. Recuperado de <http://esource.dbs.ie/handle/10788/462>.

Correia, K. \& Borloti, E. (2011). Mulher e depressão: uma análise comportamental-contextual. Acta Comportamentalia, 19(3), 359-373. Recuperado de <http://pepsic.bvsalud.org/pdf/actac/v19n3/a07.pdf>. 
Daye, C., Webb, J., \& Jafari, N. (2014). Exploring self-compassion as a refuge against recalling the body-related shaming of caregiver eating messages on dimensions of objectified body consciousness in college women. Body Image, 11(4), 547-556. http://dx.doi.org/10.1016/j.bodyim.2014.08.001

Duncan, L., Coatsworth, J., \& Greenberg, M. (2009). A model of mindful parenting: implications for parent-child relationships and prevention research. Clinical Child and Family Psychology Review, 12, 255-270. http://dx.doi. org/10.1007/s10567-009-0046-3

Ferguson, L., Kowalski, K., Mack, D., \& Sabiston, A. (2014). Exploring self-compassion and eudaimonic well-being in young women athletes. Journal of Sport \& Exercise Psychology, 36(2), 203-216. http://dx.doi.org/10.1123/jsep.2013-0096

Hutz, C. S. \& Zanon, C. (2011). Revisão da adaptação, validação e normatização da Escala de Autoestima de Rosenberg. Avaliação Psicológica, 10(1), 41-49. Recuperado de <http://pepsic.bvsalud.org/pdf/avp/v10n1/v10n1a05.pdf>.

Instituto Brasileiro de Geografia e Estatística (2014). Pesquisa Nacional de Saúde. Percepção do estado de saúde, estilos de vida e doenças crônicas. Brasil, grandes regiões e unidades da federação. Rio de Janeiro: IBGE.

Jeffries, S. \& Konnert, C. (2002). Regret and psychological well-being among voluntarily and involuntarily childless women and mothers. The International Journal of Aging \& Human Development, 54(2), 89-106. http://dx.doi. org/10.2190/J08N-VBVG-6PXM-0TTN

Joshi, S., Kumari, S., \& Jain, M. (2008). Religious belief and its relation to psychological well-being. Journal of the Indian Academy of Applied Psychology, 34(2), 345-354. Recuperado de: <http://medind.nic.in/jak/t08/i2/jakt08i2p345.pdf $>$.

Judge, T. \& Bono, J. (2001). Relationship of core self-evaluations traits - self-esteem, generalized self-efficacy, locus of control, and emotional stability - with job satisfaction and job performance: a meta-analysis. Journal of Applied Psychology, 86(1), 80-92. http://dx.doi.org/10.1037/0021-9010.86.1.80

Justo, L. \& Calil, H. (2006). Depressão - o mesmo acometimento para homens e mulheres? Revista de Psiquiatria Clínica, 33(2), 74-79. Recuperado de <http://urutu.honet.usp.br/ipq/revista/vol33/n2/74.html>.

Liss, M. \& Erchull, M. (2015). Not hating what you see: self-compassion may protect against negative mental health variables connected to self-objectification in college women. Body Image, 14, 5-12. http://dx.doi.org/10.1016/j. bodyim.2015.02.006

Maltby, J., Lewin, C., \& Day, L. (1999). Religious orientation and psychological well-being: the role of the frequency of personal prayer. British Journal of Health Psychology, 4, 363-378. http://dx.doi.org/10.1348/135910799168704

Mosewich, A., Crocker, P., Kowalski, K., \& Delongis, A. (2013). Applying self-compassion in sport: an intervention with women athletes. Journal of Sport \& Exercise Psychology, 35(5), 514-524. Recuperado de <http://www.humankinetics. com/acucustom/sitename/Documents/DocumentItem/07_Mosewich_JSEP_0008.pdf $>$.

Mosewich, A., Kowalski, K., Sabiston, C., Sedgwick, W., \& Tracy, J. (2011). Self-compassion: a potential resource for young women athletes. Journal of Sport \& Exercise Psychology, 33(1), 103-123. Recuperado de <http://citeseerx. ist.psu.edu/viewdoc/download;jsessionid=127CE050D9EFC70A3644206271C526BD?doi=10.1.1.383.7878\&rep= rep1\&type $=$ pdf $>$.

Neely, M., Schallert, D., Mohammed, S., Roberts, R., \& Chen, Y.-J. (2009). Self-kindness when facing stress: the role of self-compassion, goal regulation, and support in college students' well-being. Motivation and Emotion, 33, 88-97. http://dx.doi.org/10.1007/s11031-008-9119-8

Neff, K. (2003a). Self-compassion: an alternative conceptualization of a healthy attitude toward oneself. Self and Identity, 2, 85-101. http://dx.doi.org/10.1080/15298860390129863

Neff, K. (2003b). The development and validation of a scale to measure self-compassion. Self and Identity, 2, 223-250. http://dx.doi.org/10.1080/15298860390209035

Neff, K., Hsieh, Y.-P., \& Dejitterat, K. (2005). Self-compassion, achievement goals, and coping with academic failure. Self and Identity, 4, 263-287. http://dx.doi.org/10.1080/13576500444000317

Neff, K. \& Lamb, L. (2009). Self-Compassion. In S. Lopez (Org.). The Encyclopedia of Positive Psychology (pp. 864-867). Malden, MA: Wiley-Blackwell.

Neff, K. \& McGehee, P. (2010). Self-compassion and psychological resilience among adolescents and young adults. Self and Identity, 9, 225-240. http://dx.doi.org/10.1080/15298860902979307

Neff, K. \& Pommier, E. (2013). The relationship between self-compassion and other-focused concern among college undergraduates, community adults, and practicing meditators. Self and Identity, 12(2), 160-176. http://dx.doi.org/10 $.1080 / 15298868.2011 .649546$

Neff, K. \& Vonk, R. (2009). Self-compassion versus global self-esteem: two different ways of relating to oneself. Journal of Personality, 77(1), 23-50. http://dx.doi.org/10.1111/j.1467-6494.2008.00537.x

Pacico, J. C., Ferraz, S. B., \& Hutz, C. S. (2014). Autoeficácia - yes we can! In C. S. Hutz (Org.). Avaliação em psicologia positiva (pp. 111-120). Porto Alegre: ArtMed.

Phillips, W. \& Ferguson, S. (2013). Self-compassion: a resource for positive aging. Journals of Gerontology Series B: Psychological Sciences and Social Sciences, 68(4), 529-539. http://dx.doi.org/10.1093/geronb/gbs091

Raes, F. (2011). The effect of self-compassion on the development of depression symptoms in a non-clinical sample. Mindfulness, 2, 33-36. http://dx.doi.org/10.1007/s12671-011-0040-y

Reis, N., Kowalski, K., Ferguson, L., Sabiston, C., Sedgwick, W., \& Crocker, P. (2015). Self-compassion and women athletes' responses to emotionally difficult sport situations: an evaluation of a brief induction. Psychology of Sport \& Exercise, 16, 18-25. http://dx.doi.org/10.1590/S1415-790X2010000400007 
Schoenefeld, S. \& Webb, J. (2013). Self-compassion and intuitive eating in college women: examining the contributions of distress tolerance and body image acceptance and action. Eating Behaviors, 14(4), 493-496. http://dx.doi. org/10.1016/j.eatbeh.2013.09.001

Shapira, L. \& Mongrain, M. (2010). The benefits of self-compassion and optimism exercises for individuals vulnerable to depression. The Journal of Positive Psychology, 5(5), 377-389. http://dx.doi.org/10.1080/17439760.2010.516763

Souza, L. K. de \& Hutz, C. S. (2016a). Adaptation of the Self-Compassion Scale for use in Brazil: evidences of construct validity. Temas em Psicologia, 24(1), 159-172. http://dx.doi.org/10.9788/TP2016.1-11

Souza, L. K. de \& Hutz, C. S. (2016b). Self-compassion in relation to self-esteem, self-efficacy and demographical aspects. Paideia, 26(64), 181-188. http://dx.doi.org/10.1590/1982-43272664201604

Twenge, J. \& Campbell, W. (2001). Age and birth cohort differences in self-esteem: a cross-temporal meta-analysis. Personality and Social Psychology Review, 5(4), 321-344. http://dx.doi.org/10.1207/S15327957PSPR0504_3

Van Dam, N., Sheppard, S., Forsyth, J., \& Earleywine, M. (2011). Self-compassion is a better predictor than mindfulness of symptom severity and quality of life in mixed anxiety and depression. Journal of Anxiety Disorders, 25, 123-130. http://dx.doi.org/10.1016/j.janxdis.2010.08.011

Wasylkiw, L., MacKinnon, A., \& MacLellan, A. (2012). Exploring the link between self-compassion and body image in university women. Body Image, 9(2), 236-245. http://dx.doi.org/10.1016/j.bodyim.2012.01.007

Wei, M., Liao, K., Ku, T.-Y., \& Shaffer, P. (2011). Attachment, self-compassion, empathy, and subjective well-being among college students and community adults. Journal of Personality, 79(1), 191-221. http://dx.doi.org/10.1111/ j.1467-6494.2010.00677.x

Yarnell, L., Stafford, R., Neff, K., Reilly, E., Knox, M., \& Mullarkey, M. (2015). Meta-analysis of gender differences in self-compassion. Self and Identity, 14(5), 499-520. http://dx.doi.org/10.1080/15298868.2015.1029966

Autores:

Luciana Karine de Souza - Doutora, Universidade Federal do Rio Grande do Sul.

Claudio Simon Hutz - Doutor, Universidade Federal do Rio Grande do Sul.

Endereço para correspondência:

Luciana Karine de Souza

Instituto de Psicologia - PPG-Psicologia

Rua Ramiro Barcelos, 2600, térreo

90035-003 Porto Alegre, RS, Brasil

<lukarides@gmail.com>

Recebido em: 29.06 .2015

Aceito em: 29.01.2016 